\title{
Comparación de alternativas para mejora de la inyección cíclica de vapor mediante simulación numérica
}

\author{
Romel Pérez ${ }^{1 *}$, Jorge Sandoval ${ }^{1}$, Carolina Barbosa ${ }^{2}$, Claudia Delgadillo ${ }^{1}$, Marta Trujillo ${ }^{1}$, Laura Osma $^{2}$, \\ Jesús Botett², Luis García ${ }^{2}$, Héctor Rodríguez ${ }^{3}$
}

${ }^{1}$ Instituto Colombiano del Petróleo (ICP). Vía Piedecuesta Km 7. Piedecuesta, Colombia

${ }^{2}$ Universidad industrial de Santander (UIS). Carrera 27 Calle 9. Bucaramanga, Colombia

${ }^{3}$ Ecopetrol S.A. - Enhanced Oil Recovery Management, Bogotá, Cundinamarca, Colombia

*E-mail: romel.perez@ecopetrol.com.co

\begin{abstract}
Resumen
La inyección cíclica de vapor (CSS, por sus siglas en inglés) es un método de recobro mejorado (EOR) térmico de los más aplicados a nivel mundial; sin embargo, presenta desafíos relacionados con la eficiencia energética del proceso y costos asociados a su operación. En el caso de Colombia, la CSS se viene aplicando en diferentes campos de crudo pesado desde hace muchos años, encontrándose en algunos casos la necesidad de implementar y combinar tecnologías para extender el límite técnico económico de dicho proceso de recobro mejorado. En ese sentido, este trabajo se fundamenta en analizar diversas alternativas que permitan mejorar la eficiencia energética y la recuperación de petróleo de forma rentable. Para llevar a cabo el análisis, se construyó un modelo de simulación numérica semi-conceptual, representativo de un campo colombiano de crudo pesado, en el cual se realizó la simulación de escenarios de producción en frío, inyección cíclica de vapor, calentamiento en fondo de pozo y la combinación del vapor con diferentes sustancias (métodos híbridos de inyección de vapor); estos resultados, evaluados bajo condiciones similares en todos los casos, permiten establecer el potencial de las tecnologías y enfocar de esta forma los esfuerzos investigativos en aquellas que se consideren más promisorias. Adicionalmente, se realizó un estudio preliminar de visualización de tecnologías de generación de vapor a través de energía solar y algunas aplicaciones en campo relevantes, debido a los desafíos de disponibilidad de combustible y altos costos asociados a la generación de vapor convencional, esto alineado con los planes de Ecopetrol del uso de tecnologías limpias. Para el caso particular de las tecnologías de calentamiento de fondo de pozo en sus diferentes modalidades (calentamiento electro-resistivo, electro-inductivo o recirculación de vapor), se evaluó el comportamiento en el modelo a través de un pozo calentador (heater well), lo que permitió definir después de varias sensibilidades que este tipo de tecnologías resultan de mayor beneficio e impacto en pozos nuevos, es decir, previo a procesos de inyección cíclica de vapor.Por otra parte, las tecnologías híbridas de inyección cíclica de vapor evaluadas, corresponden a la adición de espumas, solventes, gases de combustión (flue gas) y nano partículas; todos con características particulares tanto en interacción con el vapor y el yacimiento, como en la forma específica de ser representados en el modelo de simulación. Para realizar la comparación se estableció el mismo ciclo de aplicación para todas las tecnologías; sin embargo, los resultados demuestran que la eficiencia de su desempeño depende fuertemente de las condiciones del yacimiento al momento de su aplicación. De acuerdo a los resultados obtenidos de la simulación numérica, en todos los casos se obtienen incrementos en la producción de petróleo con valores particulares para cada una de las tecnologías evaluadas. Se recomienda continuar con estudios detallados tanto a nivel experimental como de ingeniería de yacimientos que permitan definir viabilidad de posibles aplicaciones en campo.
\end{abstract}

Palabras clave: Recobro mejorado, Inyección cíclica de vapor, tecnologías híbridas y simulación numérica.

\section{Comparison of alternate technologies to improve cyclic steam stimulation through numerical simulation}

\begin{abstract}
Cyclic steam stimulation (CSS), is the most applied thermal enhanced oil recovery (EOR) method worldwide. However, despite the vast experience gained over the last few decades CSS still have its challenges including but not limited to energy efficiency and operational costs. CSS has been evaluated for several years in Colombian heavy oil reservoirs. With CCS

Cita: Pérez, R., Sandoval, J., Barbosa, C., Delgadillo, C., Trujillo, M., Osma, L., Botett, J., García, L. \& Rodríguez, H. (2018). Comparación de alternativas para mejora de la inyección cíclica de vapor mediante simulación numérica. Revista Fuentes: El reventón energético, 16(2), 91-108.
\end{abstract}


approaching its maturity new alternatives and injection strategies are required to potentially extend the technical-economical limit of this recovery process. Therefore, this work is focused on the evaluation of different strategies to potentially improve CCS performance. Alternate methods evaluated range from steam generation with solar energy to well heating strategies and CCS combined with flue gas, foams, solvents and nano particles (hybrid CCS methods). This evaluation was carried out using a semi-conceptual numerical simulation model representative of a Colombian heavy oil reservoir currently under CCS. The performance of hybrid CCS methods were compared against cold production, conventional CSS (base case) and heating well technologies. Furthermore, this paper summarizes the visualization of steam generation technologies through solar energy and some relevant field applications. Downhole heating technologies (i.e. electro-resistive heating, electro-inductive heating or steam recirculation) were evaluated using the "heater well" option. On the other hand, the hybrid CCS methods evaluated considered specific parameters and injection schedules for each technology to represent as much as possible the performance documented in the literature including some field applications. Based on the DNI (Direct Normal Irradiance), some regions of Colombia are candidates to consider steam generation based on solar energy. Despite the cost and environmental benefits, high CAPEX and the requirement of large acreage were some of the constraints identified. Regarding the simulation study, preliminary results of this study suggest that heating technologies showed greater performance in new wells and before CCS start (Pre-heating approach at initial reservoir pressure). Regarding the hybrid CSS methods, the efficiency of each of the technologies evaluated strongly depends on the reservoir conditions (CCS maturity) at the time it is implemented. Specifically, CCS combined with solvents or nano particles showed better performances at early stages of CSS (i.e. $2^{\text {nd }}$ steam cycle). On the contrary, CCS combined with foams and flue gas evidenced better efficiencies when implemented at later stages of CSS (i.e. $6^{\text {th }}$ steam cycle). Despite the potential identified in some of the technologies evaluated develop detailed experimental and simulation studies are strongly recommended. Finally, the approach followed in this study represents a reasonable screening methodology to preliminary support decision-making strategies of technical teams and managers identifying most promising technologies based on the resources available and current conditions of Colombian heavy oil reservoirs.

Keywords: Enhanced oil recovery (EOR), cycle steam stimulation, hybrid technologies and numerical simulation

\section{Introducción}

La inyección de vapor es el método de recobro mejorado de mayor aplicación y éxito en campos de crudo pesado y extra pesado a nivel mundial. Sandrea y Dharod (2016) reportan que el $40 \%$ de los proyectos activos de recobro mejorado (EOR) son por inyección de vapor. Se estima que el $23 \%$ de la producción global de proyectos de EOR proviene de métodos térmicos dominado por la inyección de vapor (SBI Energy, 2010). Sin embargo, la eficiencia de este método de recobro puede verse afectada por diversos factores que deben mitigarse a través de la implementación de nuevas tecnologías o de estrategias de gerenciamiento de energía.

En Colombia existen diversas aplicaciones de procesos de inyección de vapor. Los campos identificados como candidatos para esta tecnología presentan alto potencial de reservas (más de 6 billones de barriles), los cuales corresponden principalmente a campos del Valle Medio del Magdalena (Naranjo, 2015).

Dichas áreas han sido desarrolladas utilizando principalmente la inyección cíclica de vapor. Sin embargo, algunas zonas están alcanzado su límite técnico-económico por lo que es necesario identificar nuevas tecnologías de recobro térmico que puedan ser implementadas y optimizadas (Díaz, 2017). Además, se están desarrollando pilotos de inyección continua de vapor en el país, lo que evidencia el interés en estos procesos y el crecimiento a nivel nacional de este tipo de tecnologías de recobro mejorado.

En muchos proyectos, los costos asociados con la generación de vapor impiden su implementación en campo por lo que adicionalmente, se presenta una visualización de oportunidades para Colombia en cuanto al uso de energías limpias para la recuperación de hidrocarburos pesados utilizando fuentes alternativas como la energía solar.

Adicionalmente, el uso de tecnologías de calentamiento en fondo de pozo, como el calentamiento eléctrico resistivo, electro inductivo y recirculación de vapor, se presentan como una alternativa a la aplicación de la inyección cíclica de vapor en áreas no calentadas y pozos nuevos. Sumado a lo anterior, si se estudia su aplicación en combinación con generación de energía de formas alternativas, como la energía solar, su aplicación podría llegar a ser competitiva.

Por otra parte, el uso de tecnologías híbridas ha sido de interés para extender la vida de los proyectos de inyección de vapor, y al mismo tiempo mejorar su rendimiento. Existen diversos tipos de aditivos al vapor, entre los cuales se encuentran las espumas, solventes, gases, nanopartículas, entre otros, que permiten optimizar el proceso y generar un incremento en la producción de petróleo. 
Las espumas han sido utilizadas para mejorar la eficiencia de la inyección de vapor desde finales de la década de 1970 y su aplicación ha sido un éxito en varios campos. El principio de la espuma es reducir la movilidad del vapor, incrementar el barrido y reducir las pérdidas de calor. Las espumas se forman mediante la inyección conjunta de vapor de agua y un tensoactivo, por lo que las burbujas de gas entran en el medio poroso a través de los poros y gargantas y ocasionan un efecto de bloqueo (Delamaide, Cuenca \& Chabert, 2016).

El uso de solventes en crudos pesados también data de los años 1970 y su uso en conjunto con el vapor surge por la intención de obtener las ventajas como: reducción de la cantidad de vapor requerido, menor consumo de gas y reducción de las emisiones de gases efecto invernadero (Morales, Patiño, Navarro y Castelblanco, 2015). Sin embargo, esto dependerá de la cantidad de gas atrapado en el yacimiento durante el ciclo de producción.

La técnica consiste en inyectar un solvente vaporizado en conjunto con el vapor y el mecanismo responsable del recobro adicional es la creación de un banco rico de solventes en frente del vapor, lo que producirá una reducción adicional de la viscosidad adicional. Los solventes utilizados son mezclas de varios componentes puros, en la mayoría de los casos refinados del petróleo (Bayestehparvin, Ali \& Abedi, 2017).

En la inyección de vapor con flue gas, cuyos principales componentes son $80-85 \% \quad \mathrm{~N}_{2}$ y $15-20 \% \quad \mathrm{CO}_{2}$; el nitrógeno tiene el efecto de aumentar la energía del yacimiento y proporcionar aislamiento térmico y el $\mathrm{CO}_{2}$ tiene el efecto de disolución, reducción de la viscosidad y expansión volumétrica (Franco, Cardona, Lopera, Mejía, \& Cortés, 2016).

Tomando en cuenta que los gases de escape del generador tienen una temperatura lo suficientemente alta, su inyección conduce a un aumento de la eficiencia de la utilización de la energía térmica. Una ventaja de este método es que permite el secuestro de una fracción de los gases emitidos, contribuyendo potencialmente con una reducción en la emisión de gases.

Para el caso particular de nanopartículas, esta es una tecnología de aplicación reciente en la industria petrolera, y combinada con el vapor tiene varios efectos, siendo el principal la mejora en la recuperación de hidrocarburos a través de la reducción de viscosidad por medio de diferentes mecanismos (Franco, et al, 2016).

Esta investigación describe el proceso de simulación numérica de tecnologías de calentamiento en fondo de pozo y tecnologías hibridas de inyección cíclica de vapor, así como una valoración de la energía solar como alternativa para la generación de vapor; todo esto con el objetivo de comparar las alternativas para mejorar la eficiencia térmica del proceso.

\section{Alternativas de mejora para la inyección cíclica de vapor}

En el desarrollo de los procesos de recobro mejorado, se han propuesto mejoras que permitan ampliar su rango de aplicación o mejorar su desempeño (Aya y Navarro, 2009). El desarrollo de este trabajo está enfocado en la valoración comparativa de alternativas de mejora de la eficiencia de la inyección cíclica de vapor.

Se busca mejorar el desempeño de la CSS en Colombia, ya sea aprovechando las energías alternativas para la generación del vapor, usando tecnologías de calentamiento de fondo de pozo como reemplazo de la CSS o modificando los fluidos inyectados con adiciones que mejoren su desempeño.

Para esta evaluación se construyó un modelo de simulación que represente el comportamiento de la CSS en un campo colombiano y se evaluaron de esta forma todas las tecnologías. A continuación se describe el modelo usado y los resultados obtenidos para cada una de las tecnologías estudiadas.

\section{Modelo base: Inyección cíclica de vapor}

Para la construcción del modelo base se usó un software comercial en su interfaz especial para simulación de procesos térmicos. Está construido en una malla de tipo radial de 20 x 5 x 94 (divisiones en "r", divisiones "theta" y dirección vertical respectivamente), con un total de 9400 bloques que equivalen a un área promedio de 5 acres. Los tamaños de celda en $r$ varían desde 0,1 a $67 \mathrm{ft}$ aumentando hacia la parte externa. El modelo tiene un solo pozo, el cual se encuentra perforado de forma vertical en todas las capas, tal como se muestra en la figura 1.

A la malla de simulación se le asignaron las propiedades promedio correspondientes a las arenas de interés del campo en estudio. Las cuales se muestran en la tabla 1. 


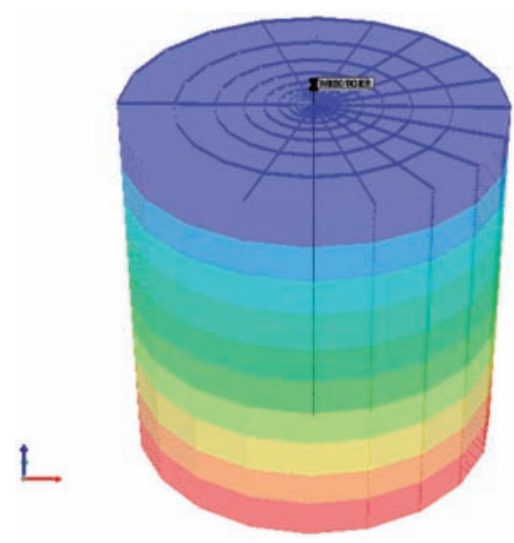

Figura 1. Modelo de simulación.

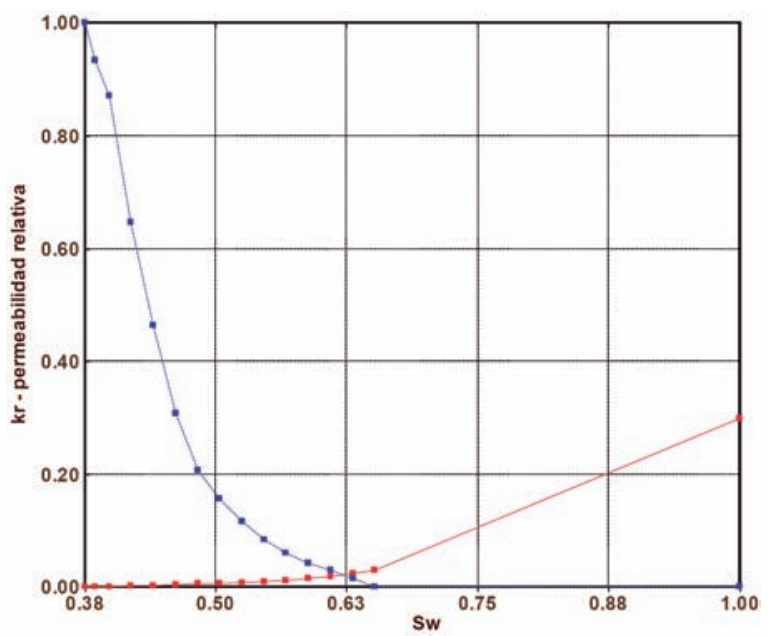

(a)
Tabla 1. Propiedades promedio campo de estudio.

\begin{tabular}{|l|c|}
\hline Porosidad (\%) & 23 \\
\hline Permeabilidad (mD) & 1170 \\
\hline Relación Kv/Kh & 0,3 \\
\hline Profundidad (ft) & $850-1320$ \\
\hline Presión Inicial (psi) & 670 \\
\hline
\end{tabular}

El modelo de fluidos cuenta con tres componentes básicos representando aceite muerto, gas en solución y agua. Se trabajó con un solo tipo de roca, cuyas curvas de permeabilidad relativa líquido-gas y agua- aceite se muestran en la figura 2.

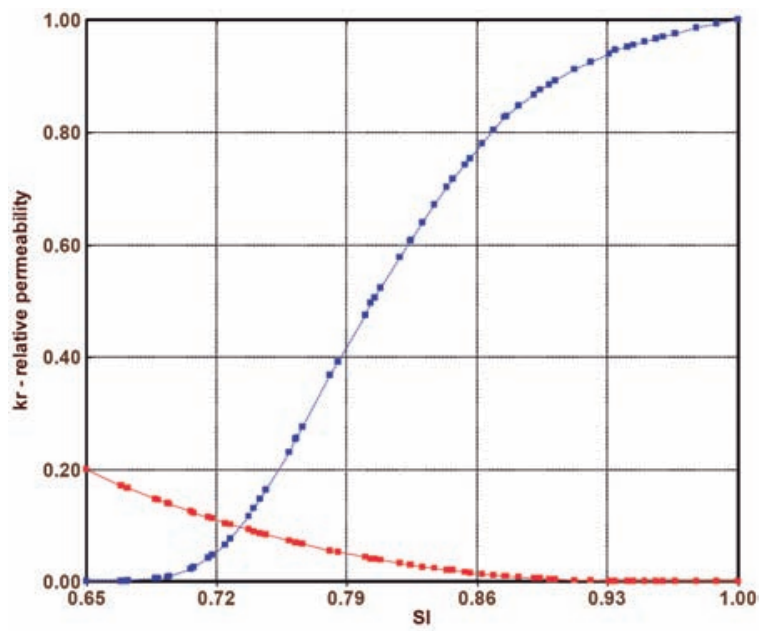

(b)

Figura 2. Curvas de permeabilidad relativa: (a) Kr agua-aceite, (b) Kr Líquido-gas.

Durante la producción primaria, según la caída de presión y la tasa diaria de producción de aceite, el modelo representa el comportamiento actual del campo de estudio.

Para el caso base de inyección cíclica de vapor, el pozo se deja en producción primaria tres meses. Después se inyecta vapor a una tasa constante de $1350 \mathrm{BWE} / \mathrm{d}$ por 6 días, con una calidad del $60 \%$ y una temperatura de inyección de $520^{\circ} \mathrm{F}$. Posteriormente, el pozo se deja 3 días de remojo y después es abierto a producción hasta que ésta alcanza la tasa de producción primaria, momento en el cual se inicia con un nuevo ciclo.

Como se observa en la figura 3, la duración de los ciclos, para el caso de los primeros varía entre 2 años y hasta 4 meses para los últimos, esto en un período de evaluación de 10 años, en el cual se alcanzan doce ciclos. Se seleccionó este periodo de evaluación debido a que después de este tiempo la duración de los ciclos disminuyó incluso hasta estar por debajo de los dos meses, por lo cual se determinó que después de este tiempo no era ni técnica ni económicamente viable continuar con el proceso.

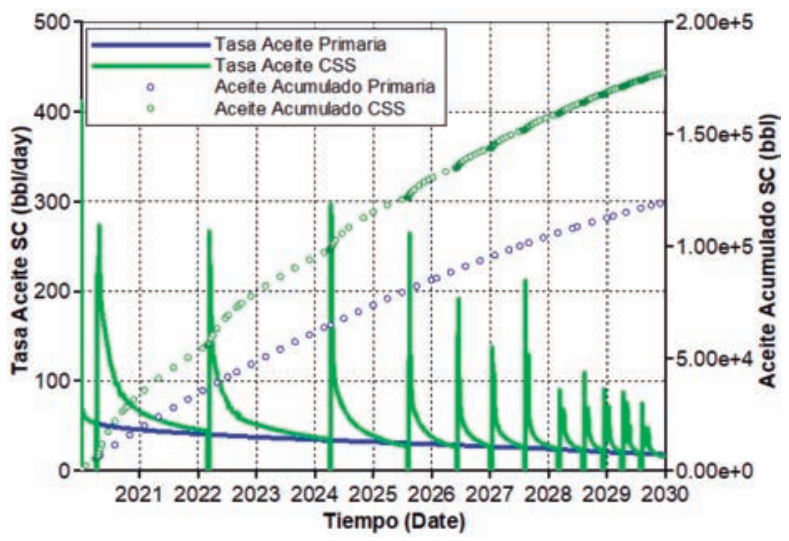

Figura 3. Producción diaria y acumulada. Caso base inyección cíclica vs producción primaria. 
El recobro obtenido por medio de producción primaria es de $11,7 \%$, equivalente a 119.627 bbl recuperados en 10 años; el recobro incremental obtenido por la implementación de inyección cíclica es de 57.668 bbl, lo que equivale a un factor de recobro incremental de casi un 6\%. Este porcentaje se obtuvo con la inyección de un total de 30.466,4 MMBTU a una tasa promedio de 423,47 MMBTU diarios por ciclo. El SOR (Steam Oil Ratio, por sus siglas en inglés) obtenido es de 0,54 $\mathrm{bbl} / \mathrm{bbl}$, valor que se encuentra dentro del rango de los valores encontrados en la literatura para inyección cíclica de vapor.

\section{Alternativa para la generación de vapor}

Para el desarrollo de proyectos de inyección de vapor en Colombia se usan generadores de vapor de un solo paso OTSG (One Through Steam Generator, por sus siglas en inglés), alimentados con agua, combustible y aire; por lo tanto, su precio y disponibilidad impactan la evaluación económica de los proyectos y el uso de combustible genera afectación ambiental.

Los proyectos de inyección de vapor ubicados en áreas con abundante luz solar pueden explotar la energía solar para generar vapor, sustituyendo el uso del gas. Usando espejos, la luz del sol se puede concentrar y usar para calentar el agua que fluye dentro de una tubería a la temperatura del vapor a alta presión. Sin embargo, este vapor generado por la energía del sol solo está disponible durante el día, provocando un ciclo día-noche en la tasa de vapor, y también se ve afectado por las variaciones estacionales en la energía solar, lo que provoca un ciclo de verano-invierno en la tasa de vapor. (Van Heel, Van Wunnik, Bentouati, \& Terres, 2010).

La medición de luz solar se expresa con base en el concepto de irradiación normal directa (DNI, por sus siglas en inglés). Esta se define como la cantidad de rayos que provienen de una línea recta del sol por área unitaria; teniendo en cuenta que ésta área siempre se mantiene perpendicular a la dirección del sol, en su posición actual en el cielo (Palmer \& Bernard, 2014).

Este recurso energético (DNI) es medido alrededor del mundo. Los lugares con valores más altos son los desiertos de Omán que reciben más de $2.000 \mathrm{KWh} /$ $\mathrm{m}^{2} /$ año, Amal recibe $2057 \mathrm{KW} / \mathrm{m}^{2}$ /año y Kuwait entre 1750/1930 KW/m²/año. (Palmer et al., 2014). Para el caso de Colombia, en la figura 4 se muestra un mapa del país con las mediciones de DNI (Lesage, \& Van de Graaf, 2016). En algunas regiones de Colombia los valores de DNI se estiman convenientes para poder implementar la generación de vapor solar, regiones como puerto Berrio
(Antioquia) puede recibir en promedio $1.579 \mathrm{KW} / \mathrm{m}^{2 /}$ año y Castilla la Nueva (Meta) $1.324 \mathrm{KWh} / \mathrm{m}^{2} /$ año.

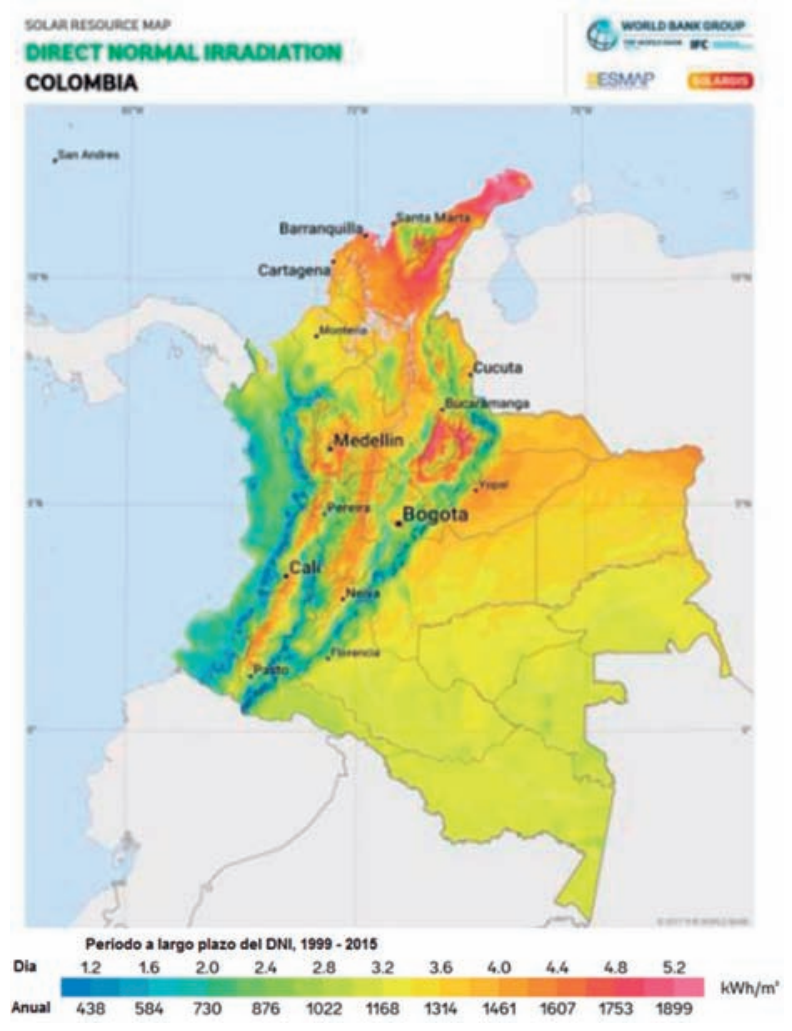

Figura 4. (C) 2017 The World Bank, Solar resource data: Solargis. Mediciones de DNI en Colombia.

Para capturar la energía solar se usan colectores, estos son espejos que concentran la irradiación solar en un solo punto o en una línea, pueden ser de diferentes tipos (plato parabólico, canal parabólico, torre solar o lineal Fresnel) (Ambrosson, \& Selin, 2016). Estos colectores deben ser ubicados de forma contigua para que se pueda capturar la mayor cantidad de rayos solares.

La cantidad de energía que se puede generar depende del DNI del lugar y de la cantidad de colectores instalados en la zona, lo que es directamente proporcional al área disponible para su instalación y a su costo.

Una de las mayores aplicaciones en campo es la planta solar Miraah ubicada en el campo Amal en Oman. Esta planta está compuesta de 36 granjas solares (Encloused Trough, tipo invernadero) en un área de 740 acres y genera aproximadamente 1,021 MW. Con esto se ahorran 5.6 trillones de BTU de gas natural cada año y se reducen las emisiones de efecto invernadero en 300000 toneladas de $\mathrm{CO}_{2}$ por año (Ab Kadir, Rafeeu, \& Adam, 2010). 
Adicionalmente, los costos de inversión para estos proyectos de acuerdo a su capacidad instalada son elevados (Sigworth, Horman, \& Knowles, 1983) y el espacio físico requerido para su instalación puede ser también un impedimento, ya que para generar una cantidad similar de energía que un generador convencional es necesario instalar colectores en un área aproximada de 20,5 Acres.

Yegane, Ayatollahi, Bashtani \& Romero (2015) refieren que el costo de la tonelada de vapor es de USD \$17 si es solar y USD \$27 si es convencional. Usando estos datos para analizar los resultados del caso base descrito anteriormente, durante 10 años de inyección cíclica de vapor la energía inyectada acumulada fue 30.466,4 MMBTU. Por lo tanto, el costo de generación de esta energía de forma convencional asciende a USD $\$ 373.823$ y si se genera con energía solar esta misma energía total inyectada costaría USD \$235.200. Sin embargo, se debe disponer de un área para instalar los colectores, mucho mayor que el área que ocupa el generador convencional.

Por todo lo anterior, es posible combinar las dos formas de generación de vapor, esto con el objetivo de disminuir los costos y el impacto ambiental; adicionalmente, es posible usar la fuente solar para la generación de energía eléctrica con el objetivo de calentar resistencias en fondo de pozo.

\section{Calentamiento en fondo de pozo}

Como opción alterna a la inyección cíclica de vapor, se presentan tres tecnologías: calentamiento electroresistivo, electroinductivo y recirculación de vapor. Este tipo de tecnologías calientan el radio del pozo, no inyectan ninguna clase de fluido o generan un incremento de presión significativo en el yacimiento y su objetivo es disminuir la viscosidad del crudo facilitando así su movimiento hacia los pozos productores.

Según Randy (2015), el calentamiento eléctrico se refiere a cualquier proceso que use la excitación molecular, convirtiendo energía eléctrica en calor, esto se hace creando una diferencia de potencial eléctrico en un elemento de baja conductividad y se genera corriente eléctrica. Existen varias formas de convertir corriente eléctrica en calor: corrientes de baja frecuencia dentro de las cuales se incluye calentamiento resistivo, corrientes de frecuencia media, dentro de los cuales se incluye calentamiento inductivo y corrientes de alta frecuencia, en donde se incluye microondas y calentamiento electromagnético.
Rodríguez, Bashbush, \& Rincón (2008), sostienen que en la industria petrolera se usan dos tipos de calentadores eléctricos: los inductivos, que generan calor de acuerdo a la ley de Maxwell para aumentar la temperatura alrededor del pozo y los resistivos que generan calor de acuerdo al efecto Joule aumentando también la temperatura alrededor del pozo.

El caso de la recirculación de vapor, es el mismo proceso de inyección de vapor convencional, con la única diferencia de que el fluido no es inyectado al yacimiento, éste es recirculado en el pozo, entregando calor latente, con el mismo radio de afectación que las tecnologías anteriormente mencionadas.

La tres tecnologías de calentamiento en fondo de pozo generan el mismo efecto en el yacimiento y su rango de afectación son las cercanías de la cara del pozo; por lo tanto, la forma de modelar numéricamente estas tres tecnologías es a través de un heater well, el cual permite ajustar una temperatura y una tasa de energía máxima entregada al yacimiento. Por lo tanto, de la simulación numérica se obtiene un radio de afectación, energía inyectada y producción de aceite todo considerado igual para las tres tecnologías de calentamiento; la diferencia está en la una evaluación económica y los costos de cada una de las herramientas evaluadas.

Para el modelamiento, se ajustó una temperatura de $520^{\circ} \mathrm{F}$ (la misma temperatura de inyección del caso base de vapor) y se hizo sensibilidad a la tasa diaria de energía inyectada, iniciando con una tasa de $500 \mathrm{MMBTU} / \mathrm{d}$ y disminuyendo hasta $5 \mathrm{MMBTU} / \mathrm{d}$.

La simulación con el heater well se realizó por 6 años, momento a partir del cual la tasa de producción de aceite cae por debajo de la producción primaria en todos los casos. Se realizó una sensibilidad a la cantidad de energía inyectada, los resultados se muestran en la figura 5, en ella se puede observar que en todos los casos del heater well e incluso con las tasas más bajas, el recobro es mayor que el del caso base de inyección cíclica de vapor.

En cuanto a la cantidad de energía inyectada, se puede ver que a tasas mayores de $30 \mathrm{MMTU} / \mathrm{d}$ el yacimiento no incrementa su producción, por lo cual el recobro es el mismo que en el caso de $500 \mathrm{MMBTU} / \mathrm{d}$ (figura 5).

Para la selección de la tasa óptima del heater well, se realizó una comparación de las energías inyectadas acumuladas en todos los casos, incluyendo la del caso base de inyección cíclica de vapor, como se observa 
en la figura 6, al igual que en la gráfica de producción, independientemente de que la tasa sea más alta, después del caso de $30 \mathrm{MMBTU} / \mathrm{d}$, el yacimiento no recibe más energía.

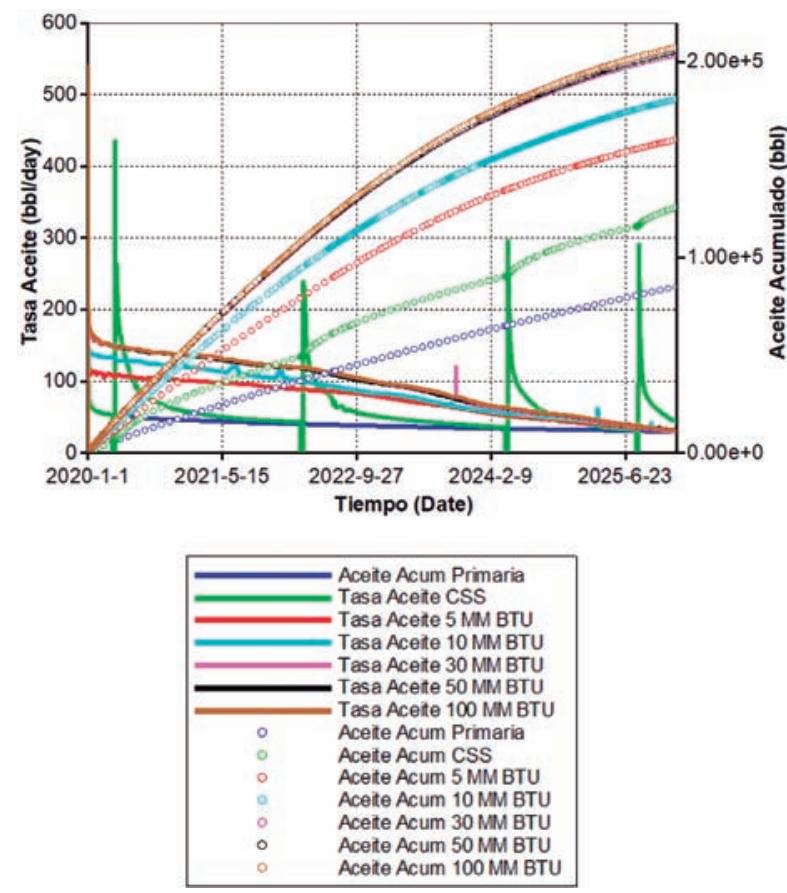

Figura 5. Sensibilidades de tasa de energía inyectada con el heater well.

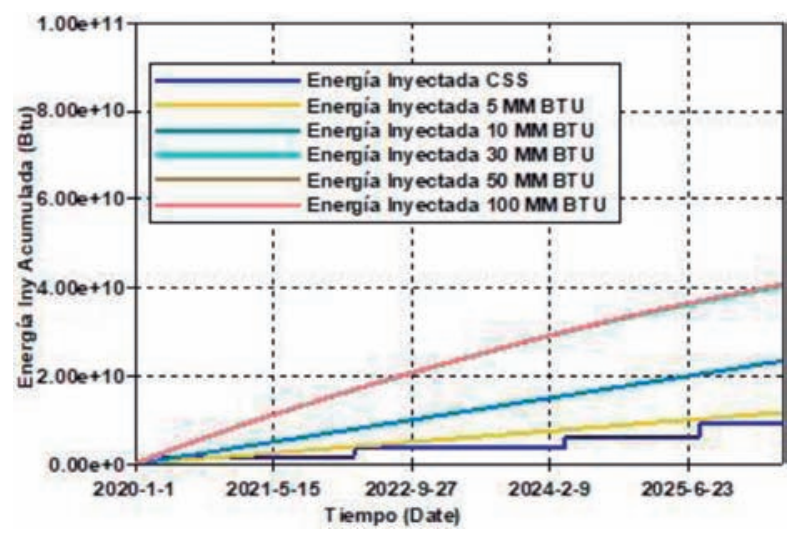

Figura 6. Escenarios del heater well: Tasas y acumulados de energía suministrada al yacimiento.

En el caso de la tasa de calentamiento de $5 \mathrm{MMBTU} / \mathrm{d}$ se aportan 10.960 MMBTU y en el caso base de inyección de vapor se inyectan 10.154 MMBTU en el total de los 6 años y medio de evaluación. Teniendo en cuenta que es importante que los valores de estas energías sean muy similares para que los casos sean comparables, se seleccionó para el heater well la tasa de $5 \mathrm{MMBTU} / \mathrm{d}$.
La figura 7 muestra los resultados de la producción de aceite acumulada y tasa diaria, comparando el caso de producción primaria con el de inyección cíclica y el heater well en el caso de 5 MMBTU de energía inyectada. Se observa que el calentamiento en fondo de pozo presenta un mejor comportamiento alcanzando el valor más alto de producción de aceite acumulada.

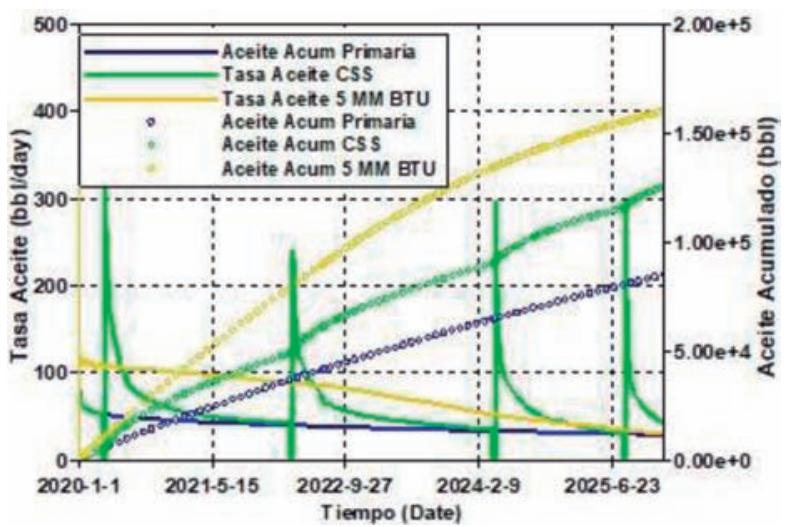

Figura 7. Comparación producción primaria, inyección cíclica y heater well.

Adicionalmente, en la tabla 2 se pueden observar los factores de recobro de los tres casos evaluados, los mismos corresponden a una producción de 134.877 bbl y 164.321 bbl para el caso base de vapor y el heater well respectivamente. Esto representa 45.672 bbl incrementales para el caso de inyección de cíclica de vapor y de $75.116 \mathrm{bbl}$ para el heater well respecto al caso de producción primaria.

Tabla 2. Resultados obtenidos a partir del uso del heater well.

\begin{tabular}{|l|c|c|c|c|}
\hline & $\begin{array}{c}\text { Factor de } \\
\text { Recobro } \\
(\%)\end{array}$ & $\begin{array}{c}\text { Aceite } \\
\text { producido } \\
\text { (bbl) }\end{array}$ & $\begin{array}{c}\text { Aceite } \\
\text { incremental } \\
\text { (bbl) }\end{array}$ & $\begin{array}{c}\text { Energía Total } \\
\text { Inyectada } \\
\text { (BTU) }\end{array}$ \\
\hline Heater Well & 16 & 164.321 & 75.116 & $1,17 \times 10^{10}$ \\
\hline CSS & 13,2 & 134.877 & 45.672 & $1,02 \times 10^{10}$ \\
\hline Primaria & 8,7 & 89.205 & --- & --- \\
\hline
\end{tabular}

Como se observa en la tabla 2, al usar una cantidad de energía similar en ambos casos (CSS y heater well), las tecnologías de calentamiento en fondo se presentan como alternativas eficientes para la explotación en etapas tempranas del campo de estudio. En este sentido, una evaluación económica permitirá decidir cuál de ellas es la más viable, dependiendo de sus requerimientos de aplicación; así como la posible implementación en conjunto con fuentes de energía alternativas (P.E. energía eólica y energía solar.) 


\section{Métodos híbridos}

Los métodos de recobro híbridos de inyección de vapor han sido desarrollados con el objetivo de aprovechar los beneficios térmicos del proceso de inyección de vapor, mejorando su desempeño con la adición de sustancias y/o fluidos que cumplan funciones específicas.

En el desarrollo de este estudio se realizaron simulaciones en el modelo base, modificando los fluidos inyectados y algunas características del modelo, todo con el objetivo de representar de forma mecanística el desempeño de la inyección de vapor con espumas, flue gas, solventes y nano partículas. Debido a que la simulación de estos procesos requiere información experimental y en algunos casos es un proceso innovador, para cada caso particular se realizaron adecuaciones que permitieran representar, de forma aproximada, su desempeño en el yacimiento.

A continuación, se describe la simulación numérica realizada, su objetivo fue comparar los procesos aplicándolos bajo las mismas condiciones. Para ello se realizaron las corridas de los métodos híbridos en el ciclo 6 del modelo; sin embargo, en algunos casos fue necesario variar el momento de aplicación, por las condiciones del proceso.

Inyección de vapor con espumas. Según Delamaide et al. (2016), la espuma ha sido utilizada para mejorar la eficiencia de la inyección de vapor desde finales de la década de 1970 y su aplicación ha sido un éxito en varios campos.

El principio de la espuma es reducir la movilidad del vapor, incrementar el barrido y reducir las pérdidas de calor. Las burbujas de gas atrapadas generadas en la espuma entran en la roca a través de los poros y gargantas y ocasionan un efecto de bloqueo, cerrando canales de flujo preferencial, haciendo el proceso más eficiente.

Para el modelamiento de la inyección de vapor con espumas se incluyeron cuatro componentes nuevos en la sección de fluidos que corresponden al surfactante, nitrógeno, lamela (corresponde a la parte líquida de la espuma) y gas atrapado (corresponde a la burbuja de gas) en la espuma con sus respectivas propiedades y cambios en la viscosidad con respecto a la temperatura. Se generaron seis reacciones básicas que representan la coalescencia de la espuma en el tiempo y por presencia de aceite, así como la regeneración de la misma.
Para la inyección de la espuma se usó el mismo pozo inyector de vapor, en él se inyecta previamente nitrógeno y posteriormente el componente que representa la espuma (mezcla de nitrógeno, agente espumante y agua) con una concentración del $0,25 \% \mathrm{vol} / \mathrm{vol}$.

Los resultados de producción se muestran en la figura 8, en donde se evidencia que el uso de la espuma extiende la duración del ciclo aproximadamente 4 meses.

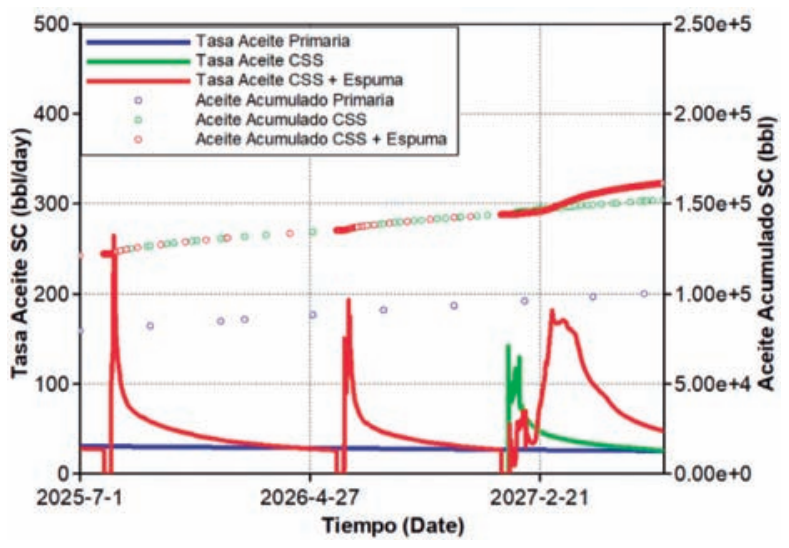

Figura 8. Resultados Inyección de vapor y espumas (ciclo 6).

Durante el ciclo de aplicación de la espuma se obtiene una producción incremental de 13.445 barriles, esto debido al bloqueo de los canales preferenciales de flujo, generado por la espuma.

Adicionalmente, el bloqueo es evidente en el comportamiento de la presión en el modelo de simulación. Como se ve en la figura 9, al inyectar la espuma hay un aumento de presión, un delta de 873 psi en el modelo de simulación, que son comparables con el delta de 852 obtenido en campo; la tendencia se mantiene, pero las presiones en el momento del inicio de la inyección son diferentes, por lo tanto los valores difieren levemente.

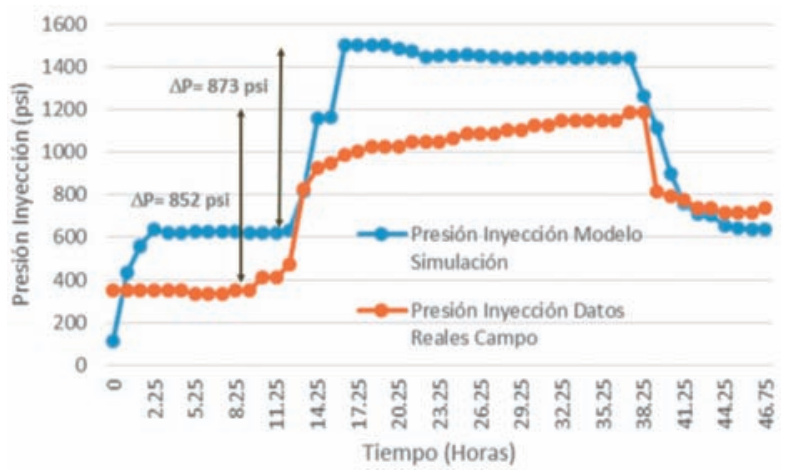

Figura 9. Presión de inyección durante la inyección de vapor con espumas. 
Con base en los resultados obtenidos, la inyección de espuma a un ciclo de vapor bajo las condiciones de presión y saturación evaluadas, sería una buena opción para bloquear los canales preferenciales de flujo, aumentar la eficiencia del proceso y por ende aumentar la producción. Sin embargo, es importante realizar una evaluación económica, ya que es necesario determinar si los costos de implementación de la tecnología lo hacen económicamente viables.

Inyección de vapor con flue gas. La inyección de vapor con flue gas se utiliza como un proceso híbrido en donde se combinen las bondades de la inyección de vapor y el desplazamiento del aceite por el efecto de los gases de combustión, también conocidos como flue gas, cuya composición química es una mezcla de nitrógeno y dióxido de carbono y otros compuestos como óxidos de azufre, óxidos de nitrógeno y monóxido de carbono en bajas proporciones.

Bajo condiciones de desplazamiento miscible, el dióxido de carbono se disuelve en el petróleo y reduce su viscosidad a la par que el nitrógeno aumenta la presión del yacimiento (De Ferrer, 2001). En el caso de la inyección inmiscible, el efecto principal de la corriente de flue gas inyectada es incrementar la presión adicionando energía, lo cual acelera el recobro de petróleo (Villaquirán, Rodríguez y Muñoz, 2017)

Para este estudio se realizó el modelamiento numérico del proceso bajo condiciones inmiscibles. Aun cuando, como en este caso, no se alcancen las presiones de miscibilidad del $\mathrm{CO}_{2}$, un porcentaje del volumen inyectado se podría llegar a solubilizar en el fluido del yacimiento. Sin embargo, los efectos e impacto en el incremento del factor de recobro de este método híbrido no han sido ampliamente documentados. La tabla 3 muestra las fracciones molares de la corriente de flue gas.

Tabla 3. Composiciones molares corriente de flue gas empleados en el estudio de simulación

\begin{tabular}{|c|c|}
\hline Componente & Valor (Fracción) \\
\hline Nitrógeno & 0,83 \\
\hline Dióxido de Carbono & 0,17 \\
\hline
\end{tabular}

Para la estimación de los volúmenes de inyección de gas se partió de que la fuente de dicho fluido fuesen los gases de chimenea que se producen en los generadores de vapor convencionales. Para ello se empleó como base de cálculo una ecuación típica de combustión en donde reaccionan el metano (suponiendo que el combustible utilizado durante la generación es gas natural), nitrógeno y oxígeno, produciéndose flue gas y agua.

Asumiendo que un mol de metano produce aproximadamente $1.000 \mathrm{BTU}$, se calculan los moles que reaccionan para producir la energía diaria inyectada del caso base, $1.350 \mathrm{BWE} / \mathrm{d}$, el valor hallado fue el máximo considerado; éste y otros intermedios se reportan en la tabla 4.

Tabla 4. Volúmenes de inyección de flue gas evaluados.

\begin{tabular}{|c|c|}
\hline Volúmenes de Inyección & Tasa de flue gas $\left(\mathbf{f t}^{3} / \mathbf{d}\right)$ \\
\hline Caso 1 & $1.017 .062,4$ \\
\hline Caso 2 & 2.000 .000 \\
\hline Caso 3 & 5.000 .000 \\
\hline Caso 4 & $6.221 .222,3$ \\
\hline
\end{tabular}

Adicionalmente, se evaluó la influencia de la variación del volumen total de inyección de flue gas sobre el recobro de aceite y sus efectos al inyectarse en conjunto con el vapor. Para ello, se consideraron cinco esquemas de inyección (tabla 5), todos diseñados de acuerdo a resultados preliminares de simulación y manteniendo fijo el volumen total final de inyección de vapor igual al del caso base.

Tabla 5. Esquemas de inyección de flue gas y vapor.

\begin{tabular}{|c|c|}
\hline \multicolumn{2}{|c|}{ Esquemas de Inyección } \\
\hline I & Coinyección \\
\hline II & 1 Día Flue gas - 6 Días Vapor \\
\hline III & 6 Días Vapor - 1 Día Flue gas \\
\hline IV & 3 Días Flue gas - 4 Días Vapor \\
\hline V & 1 Día Flue gas -6 Días Coinyección -1 Día Flue gas \\
\hline
\end{tabular}

Combinando las distintas tasas y los esquemas de inyección planteados, se generaron veinte escenarios, mostrados en la tabla 6. En ellos se mantienen las condiciones de inyección reportadas en el caso base (1350 BWE/d por 6 días, $60 \%$ calidad de vapor y temperatura de inyección de $520^{\circ} \mathrm{F}$ ), así como un volumen total de vapor inyectado similar en todos los casos, 8100 BWE.

Su aplicación se evaluó en el ciclo 6 del caso base de inyección cíclica de vapor. Los resultados de simulación muestran que los escenarios con mayor producción incremental fueron el 15 y 16; la figura 10 muestra la respuesta de producción de aceite de ellos. 
Tabla 6. Escenarios evaluados en la inyección de flue gas y vapor.

\begin{tabular}{|c|c|c|c|c|}
\hline 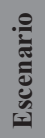 & $\begin{array}{l}\text { Esquema } \\
\text { de } \\
\text { inyección }\end{array}$ & $\begin{array}{c}\text { Tasa de flue gas } \\
\left(\mathrm{ff}^{\mathbf{3}} / \mathrm{d}\right)\end{array}$ & $\begin{array}{c}\begin{array}{c}\text { Volumen } \\
\text { total de flue } \\
\text { gas inyectado } \\
\left(\mathrm{MMft}^{3}\right)\end{array}\end{array}$ & $\begin{array}{l}\text { Producción } \\
\text { incremental } \\
\text { (bbl) }\end{array}$ \\
\hline 1 & \multirow{4}{*}{ I } & $1.017 .062,4$ & 6,1 & -- \\
\hline 2 & & 2.000 .000 & 12 & 1199 \\
\hline 3 & & 5.000 .000 & 30 & 1837 \\
\hline 4 & & $6.221 .222,3$ & 37,3 & 1910 \\
\hline 5 & \multirow{4}{*}{ II } & $1.017 .062,4$ & 1,02 & -- \\
\hline 6 & & 2.000 .000 & 2 & -- \\
\hline 7 & & 5.000 .000 & 5 & 499 \\
\hline 8 & & $6.221 .222,3$ & 6,22 & 1.102 \\
\hline 9 & \multirow{4}{*}{ III } & $1.017 .062,4$ & 1,02 & -- \\
\hline 10 & & 2.000 .000 & 2 & -- \\
\hline 11 & & 5.000 .000 & 5 & 333 \\
\hline 12 & & $6.221 .222,3$ & 6,22 & 451 \\
\hline 13 & \multirow{4}{*}{ IV } & $1.017 .062,4$ & 3,05 & -- \\
\hline 14 & & $2,000.000$ & 6 & 751 \\
\hline 15 & & 5.000 .000 & 15 & 3.564 \\
\hline 16 & & $622.122,3$ & 18,66 & 3.593 \\
\hline 17 & \multirow{4}{*}{$\mathrm{V}$} & $1.017 .062,4$ & 8,14 & -- \\
\hline 18 & & 2.000 .000 & 16 & 1.360 \\
\hline 19 & & 5.000 .000 & 40 & 2.075 \\
\hline 20 & & $6.221 .222,3$ & 49,77 & 2.218 \\
\hline
\end{tabular}

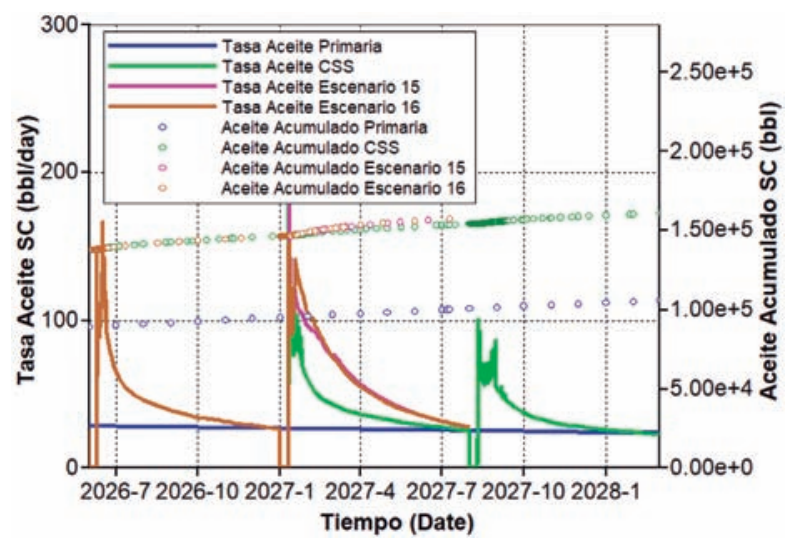

Figura 10. Resultados inyección de vapor y flue gas.

Las producciones incrementales obtenidas en los escenarios 15 y 16 alcanzaron los 3.564 y 3.593 barriles, respectivamente. Por lo tanto, la diferencia entre escenarios no es significativa y pueden considerarse dentro del error de la predicción. Adicionalmente, se identifican limitaciones en el simulador comercial empleado para capturar fenómenos de efectos de gas atrapado e histéresis de las permeabilidades relativas, dificultándose la evaluación de diferentes escenarios.
Por otra parte, si se analizan los escenarios 8, 12 y 14 (tabla 6), en ellos se inyectaron aproximadamente los mismos volúmenes de gases de combustión y vapor; sin embargo, la producción incremental es mayor en el escenario 8.

Finalmente, con los resultados se observó que a medida que aumenta el volumen de inyección de flue gas, el recobro se impacta de forma positiva. Sin embargo, los mejores escenarios no se constituyeron en aquellos en donde se inyectó el mayor volumen de gas, se presentaron casos en donde incluso la producción resultó menor a la del caso base. Por lo tanto, se observa que existe una incidencia en la forma cómo se inyecta el flue gas en conjunto con el vapor y la selección de un posible caso de aplicación en campo depende de una evaluación económica detallada.

Inyección de vapor con solventes. La inyección de vapor con solventes se presenta como una opción de mejora a la inyección de vapor convencional, como lo refieren Andarcia, Bermudez, Reyes, Caycedo \& Suarez (2014). Se espera que la presencia del solvente contribuya a disminuir la relación de vapor inyectado/ aceite producido (Steam oil ratio, SOR), esto con la selección del solvente adecuado para cada caso que permita la reducción de la viscosidad del crudo por efecto de dilución.

Por lo tanto, el objetivo de éste método híbrido es obtener los beneficios de las altas tasas de producción del método térmico y los beneficios de la disminución del volumen de vapor requerido por efecto del solvente inyectado (Morales, et al., 2015).

En la evaluación de la inyección de vapor con solventes, la mezcla de solventes considerada fue propano y butano (Colina, 2009). El primer paso en el modelamiento fue la inclusión de los nuevos componentes en la sección de fluidos del modelo base, para ello se utilizaron las constantes de partición que modelan el comportamiento de dichos solventes cuando interactúan con el fluido del yacimiento estudiado.

En este proceso de inyección de vapor con solventes, éste último se inyecta vaporizado y en este caso, se generaron escenarios en donde se varían: la relación masa-solvente/masa-vapor, las proporciones de mezcla, el volumen total de inyección y el esquema de inyección. Las variaciones para la relación masa-solvente/masavapor para la mezcla considerada como punto de partida fue de 5/100 encontrado como óptimo para el propano (Sandoval, 2004) y se variaron de acuerdo con lo descrito en la tabla 7. 
Tabla 7. Variaciones en masa consideradas.

\begin{tabular}{|c|c|}
\hline Relación en masa & Volumen de Mezcla, $\mathbf{f t}^{3} / \mathbf{d}$ \\
\hline $5 / 100$ & 203.778 \\
\hline $10 / 100$ & 407.556 \\
\hline $15 / 100$ & 611.334 \\
\hline $20 / 100$ & 815.112 \\
\hline
\end{tabular}

Las tasas diarias de inyección de mezcla de solvente fueron calculadas con base en el valor diario de inyección de vapor del caso base, $1350 \mathrm{BWE} / \mathrm{d}$ y las relaciones en volumen de mezcla consideradas fueron 1:1, 2:1 y 5:1, primando siempre el butano.

Los esquemas de inyección considerados fueron la coinyección (I) e inyección por baches (II). Esta última consistió en inyectar durante medio día vapor, seguido del volumen total de inyección de mezcla y finalizando con el volumen restante de vapor.

La cantidad de vapor inyectada fue la misma del caso base, $8100 \mathrm{BWE}$ en total, calidad del $60 \%$, condiciones de presión y temperatura iguales a las del caso base $\left(520^{\circ} \mathrm{F}\right)$ y el mismo tiempo de remojo remojo (3 días). Variando cada una de las condiciones descritas se generaron 24 escenarios de aplicación (tabla 8).

Los solventes que se inyectan al yacimiento interactúan directamente con el crudo presente, por lo tanto, este método tiene mejor desempeño en etapas tempranas de la inyección cíclica de vapor, donde la saturación de aceite es mayor; por esto, los escenarios descritos fueron evaluados en el ciclo 2 y la producción incremental obtenida se reporta en la tabla 8.

En general, los resultados obtenidos de la inyección de vapor con solventes en el ciclo 2 muestran que a medida que se aumenta el volumen total de inyección de solventes, la producción de aceite se incrementa. El escenario que obtuvo la mayor producción incremental respecto a la línea base de inyección de vapor fue el escenario 22, con 4983 bbl.

Con el objetivo de comparar las tecnologías, se replicaron en el ciclo 6 las condiciones del mejor escenario obtenido en el ciclo 2, así: relación de mezcla 5:1, relación masa-solvente/masa-vapor 20/100 y esquema de inyección a través de baches. Los resultados obtenidos de la inyección de vapor con solventes en el ciclo 6 se presentan en la figura 11, la producción incremental fue de 1.088 barriles, valor inferior al obtenido en el ciclo 2. Esto se debe a la baja saturación de aceite en etapas avanzadas después de múltiples ciclos de inyección de vapor.
Tabla 8. Condiciones escenarios y producción incremental de aceite en el ciclo 2.

\begin{tabular}{|c|c|c|c|c|c|}
\hline 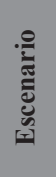 & 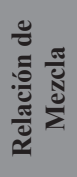 & $\begin{array}{c}\text { Tasa } \\
\text { mezcla } \\
\text { solvente } \\
\left(\mathbf{f t}^{3} / \mathbf{d}\right)\end{array}$ & $\begin{array}{c}\text { Volumen } \\
\text { total } \\
\text { solvente } \\
\text { inyectado } \\
\left(\mathbf{f t}^{3}\right)\end{array}$ & 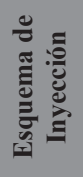 & $\begin{array}{l}\text { Producción } \\
\text { Incremental } \\
\text { Ciclo } 2 \text { (bbl) }\end{array}$ \\
\hline 1 & \multirow{2}{*}{$1: 1$} & \multirow{6}{*}{203.778} & \multirow{6}{*}{1.222 .668} & I & -- \\
\hline 2 & & & & II & 170 \\
\hline 3 & \multirow{2}{*}{$5: 1$} & & & I & 25 \\
\hline 4 & & & & II & 278 \\
\hline 5 & \multirow{2}{*}{$2: 1$} & & & I & -- \\
\hline 6 & & & & II & 280 \\
\hline 7 & \multirow{2}{*}{$1: 1$} & \multirow{6}{*}{407.556} & \multirow{6}{*}{2.445 .336} & I & 626 \\
\hline 8 & & & & II & 1.254 \\
\hline 9 & \multirow{2}{*}{$5: 1$} & & & I & 714 \\
\hline 10 & & & & II & 1.432 \\
\hline 11 & \multirow{2}{*}{$2: 1$} & & & I & 680 \\
\hline 12 & & & & II & 1.333 \\
\hline 13 & \multirow{2}{*}{$1: 1$} & \multirow{6}{*}{611.334} & \multirow{6}{*}{3.668 .004} & I & 1.536 \\
\hline 14 & & & & II & 2.691 \\
\hline 15 & \multirow{2}{*}{$5: 1$} & & & I & 1.687 \\
\hline 16 & & & & II & 3.067 \\
\hline 17 & \multirow{2}{*}{$2: 1$} & & & I & 1.598 \\
\hline 18 & & & & II & 2.877 \\
\hline 19 & \multirow{2}{*}{$1: 1$} & \multirow{6}{*}{815.112} & \multirow{6}{*}{4.890 .672} & I & 2.537 \\
\hline 20 & & & & II & 4.357 \\
\hline 21 & \multirow{2}{*}{$5: 1$} & & & I & -- \\
\hline 22 & & & & II & 4.983 \\
\hline 23 & \multirow{2}{*}{$2: 1$} & & & I & 2.770 \\
\hline 24 & & & & II & 4.723 \\
\hline
\end{tabular}

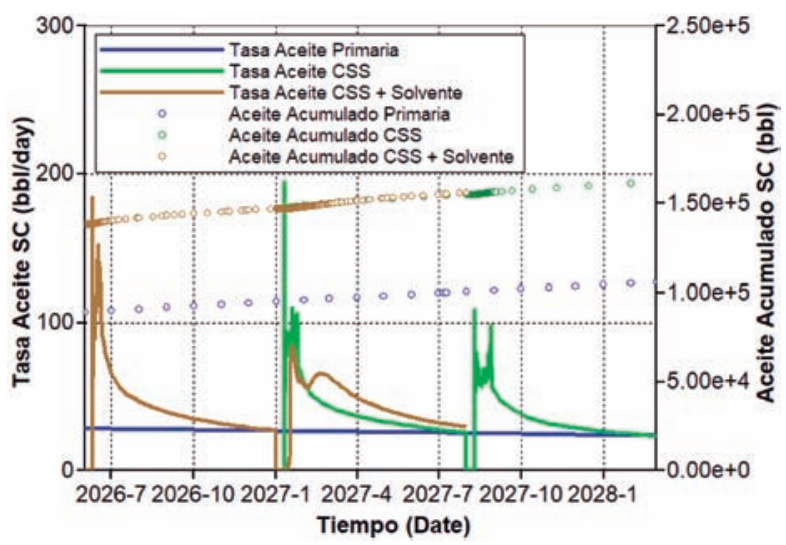

Figura 11. Resultados inyección de vapor con solventes (ciclo 6).

Por lo anterior, la inyección híbrida con solventes se considera más adecuadas en etapas tempranas de la inyección cíclica de vapor; siempre y cuando las 
economías de los proyectos lo justifiquen. En estos proyectos la recuperación del solvente inyectado (típicamente $>90 \%$ ) representa una variable crítica en este método híbrido de inyección de vapor.

Inyección de vapor con nanopartículas. HamediShokrlu (2013), plantea que además de los desafíos enfrentados durante la etapa de producción y el bajo factor de recuperación, los proyectos de producción de crudo pesado/bitumen sufren de otros problemas, incluyendo contaminación ambiental, transporte y conversión de crudo pesado/bitumen a productos de petróleo refinados. Por lo tanto, las técnicas de recuperación se deben modificar de forma que generen algún grado de mejoramiento durante la fase de producción (mejoramiento in-situ). El uso de nano partículas ha demostrado a nivel de laboratorio y en limtadas aplicaciones en campo (Maity, Ancheyta, \& Marroquín, 2010), ser un complemento técnicamente eficaz para alcanzar este objetivo.

La nanotecnología es la ciencia de los materiales en un rango muy cercano a las dimensiones moleculares (1-100 $\mathrm{nm}$ ), que ha cambiado nuestro punto de vista en muchos aspectos científicos y ha demostrado nuevas vías para potencialmente resolver viejos problemas que quedaron sin una solución sólida a través del uso de las tecnologías anteriores. Como resultado de las nuevas propiedades y la introducción de fenómenos especiales que se producen en este rango de tamaño, los materiales encuentran un potencial considerable para enfrentar los desafíos que parecían estar lejos del alcance a través de la tecnología a escala macro (Ayatollahi \& Zerafat, 2012).

En su revisión bibliográfica Hamedi-Shokrlu (2013), establece que la reducción de la viscosidad del crudo pesado/bitumen no solo se debe al efecto de alta temperatura sino también a una serie de reacciones químicas. Estas reacciones conocidas como acuatermólisis que ocurren entre los componentes de vapor, arena y aceite conducen a un mayor cambio de las propiedades físicas y químicas del crudo pesado/ bitumen. Estas reacciones pueden ser catalizadas por especies metálicas. Los principales efectos de la acuatermólisis son la reducción de asfaltenos y resinas, aumentando los saturados y aromáticos, reduciendo el peso molecular, disminuyendo el contenido de azufre, aumentando la relación $\mathrm{H} / \mathrm{C}$ y la reducción de la viscosidad del aceite producido.

Diferentes investigaciones a nivel de laboratorio han evaluado los mecanismos responsables del incremento en la producción de crudo por la aplicación de nano partículas en procesos de inyección cíclica y continua de vapor. Cómo se observa en la figura 12, uno de los mecanismos más estudiados es la reducción de la viscosidad, la cual puede estar asociada al aumento de la gravedad API, la relación H/C y a los cambios composicionales del crudo.

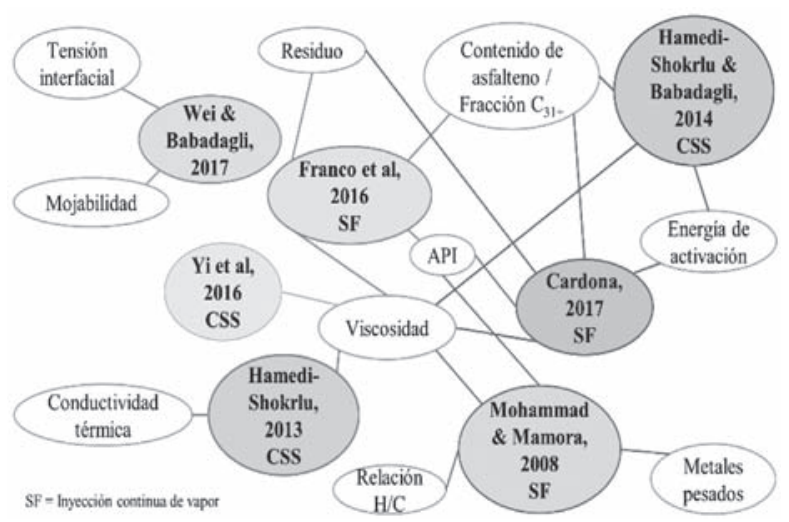

Figura 12. Principales mecanismos evaluados en la aplicación de nano partículas en inyección de vapor.

Por lo anterior, para realizar este estudio de simulación del proceso de inyección de vapor con nano partículas, en este trabajo se seleccionó este efecto como el principal mecanismo para la producción incremental de crudo. Para este fin, se introdujo una reacción que representa la interacción entre el crudo pesado, la nano partícula (inyectada en un medio acuoso) y el vapor. Básicamente, esta reacción lleva a la generación de un crudo mejorado cuyas propiedades fueron determinadas a partir de información experimental y de aplicaciones en campo, disponible en la literatura.

Para la simulación se requieren parámetros adicionales, tales como: energía de activación, factor de frecuencia, temperatura de craqueo de los asfaltenos, relación nano partícula/vapor inyectado, porcentaje de reducción de la viscosidad, concentración de nano partícula en el nano fluido, nano partícula a evaluar. Estos valores también fueron obtenidos de la literatura y se presentan en la tabla 9, junto con las propiedades del crudo mejorado.

Los resultados de la implementación de esta tecnología en el ciclo 6, bajo las condiciones presentadas en la tabla 9 , se muestran en la siguiente figura.

En la figura 13 se observa que no hay producción incremental de petróleo asociada a la adición de las nano partículas. Estos resultados se presentan debido que en esta etapa del proceso de inyección cíclica de vapor, las condiciones en el área del yacimiento que ha sido afectada, dan como resultado un potencial de producción que no es favorable para implementar el proceso con inyección de nano partículas. 
Tabla 9. Propiedades utilizadas para la simulación de nano partículas + inyección de vapor.

\begin{tabular}{|c|c|c|c|}
\hline Propiedad & \begin{tabular}{c|} 
Valor \\
en la \\
literatura
\end{tabular} & Fuente & $\begin{array}{c}\text { Valor } \\
\text { para el } \\
\text { campo } \\
\text { bajo } \\
\text { estudio }\end{array}$ \\
\hline $\begin{array}{c}\text { Peso } \\
\text { molecular } \\
\text { del crudo } \\
\text { mejorado } \\
\text { (lb/lbmol) }\end{array}$ & $\begin{array}{c}40 \% \\
\text { menos que } \\
\text { el crudo } \\
\text { original }\end{array}$ & $\begin{array}{c}\text { Wen, Zhao, Liu \& Hu, } \\
2007\end{array}$ & 243 \\
\hline $\begin{array}{c}\text { Densidad } \\
\text { del crudo } \\
\text { mejorado } \\
\left(\mathrm{lb} / \mathrm{ft}^{3}\right)\end{array}$ & $\begin{array}{c}6,05 \% \\
\text { menos que } \\
\text { el crudo } \\
\text { original }\end{array}$ & Wen et al., 2007 & 57,97 \\
\hline $\begin{array}{c}\text { Ea } \\
\text { (BTUlbmol) }\end{array}$ & 13.065 & Cardona, 2017 & 13.065 \\
\hline $\begin{array}{l}\text { Factor de } \\
\text { frecuencia } \\
\quad(1 / \mathrm{d})\end{array}$ & 1,8 & $\begin{array}{l}\text { Hamedi \& Babadagli, } \\
2014\end{array}$ & 1,8 \\
\hline $\begin{array}{c}\text { Temperatura } \\
\text { de craqueo de } \\
\text { los asfaltenos } \\
\left({ }^{\circ} \mathrm{F}\right)\end{array}$ & 410 & Cardona, 2017 & 410 \\
\hline $\begin{array}{c}\text { Relación } \\
\text { nano partícula } \\
\text { (Kmol) / vapor } \\
\text { inyectado } \\
\text { (Tonelada) }\end{array}$ & 0,02 & $\begin{array}{l}\text { Zhong, Liu, Fan, \& } \\
\text { Jiang, } 2003\end{array}$ & 0,02 \\
\hline $\begin{array}{l}\text { Porcentaje de } \\
\text { reducción de } \\
\text { la viscosidad } \\
\text { del crudo } \\
\text { original } \\
(\%)\end{array}$ & $60-80$ & $\begin{array}{c}\text { Mohammad \& } \\
\text { Mamora, 2008 / Maity } \\
\text { et al., } 2010 \text { / Hamedi- } \\
\text { Shokrlu, } 2013 \\
\text { Franco, Cardona, } \\
\text { Lopera, Mejía } \\
\text { \& Cortés, 2016 / } \\
\text { Cardona, } 2017\end{array}$ & 70 \\
\hline $\begin{array}{l}\text { Concentración } \\
\text { de nano } \\
\text { partícula en el } \\
\text { nano fluido } \\
\text { (\% peso) }\end{array}$ & $0,02-0.5$ & $\begin{array}{c}\text { Hamedi-Shokrlu, } 2014 \\
\text { / Srinivasan \& Shah , } \\
2014 \text { / Li, Genys, Wang } \\
\text { \& Torsæter, 2015 / } \\
\text { Alomair, 2016 / Tajmiri } \\
\text { \& Reza, 2016 / } \\
\text { Patel, Shah, Ahmed \& } \\
\text { Ucan, 2018 / Afzal, } \\
\text { Ehsani, Nikookar \& } \\
\text { Roayaei, 2018 }\end{array}$ & 0,085 \\
\hline $\begin{array}{l}\text { Tipo de nano } \\
\text { partícula }\end{array}$ & $\begin{array}{c}\mathrm{NiO}, \\
\mathrm{SNi}_{1} \mathrm{Pd} 1, \\
\mathrm{SiO}_{2}, \\
\mathrm{Fe}_{3}, \\
\mathrm{WO}_{3}, \\
\mathrm{ZrO}_{2}, \\
\mathrm{CuO} \\
\mathrm{ZnO} \\
\mathrm{Al}_{2} \mathrm{O}_{3}\end{array}$ & $\begin{array}{l}\text { Hamedi-Shokrlu, } 2014 \\
\text { / Srinivasan et al., } \\
2014 \text { / Alomair, } 2016 \\
\text { / Franco et al., 2016 / } \\
\text { Tajmiri \& Reza, 2016 } \\
\text { / Wei \& Babadagli, } \\
\text { 2016 / Lakhova, } \\
\text { Petrov, Ibragimova, } \\
\text { Kayukova, Safiulina, } \\
\text { Shinkarev \& Okekwe, } \\
2017 \text { / Patel et al., } \\
2018 \text { / Afzal et al., } \\
\text { 2018 / Bruns \& } \\
\text { Babadagli, } 2018\end{array}$ & $\mathrm{NiO}$ \\
\hline
\end{tabular}

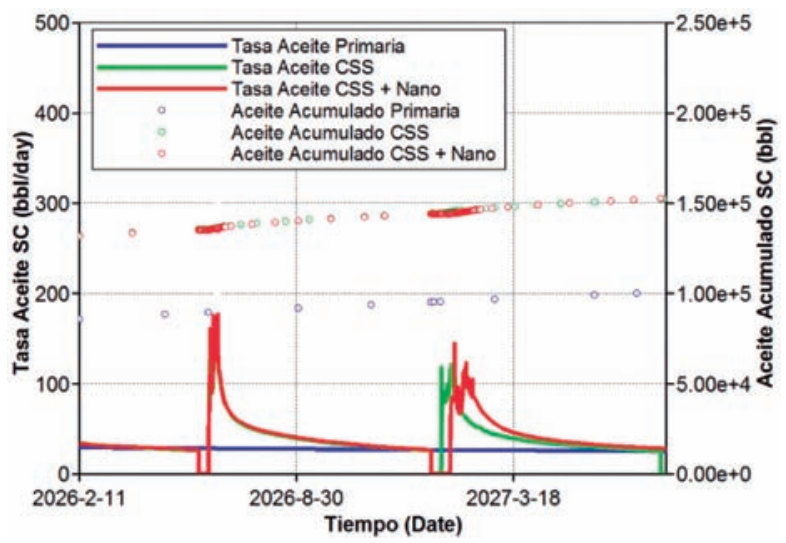

Figura 13. Resultados inyección de vapor con nano partículas (ciclo 6).

La figura 14 muestra que la presión promedio del yacimiento ha disminuido, en especial en la parte baja del modelo.

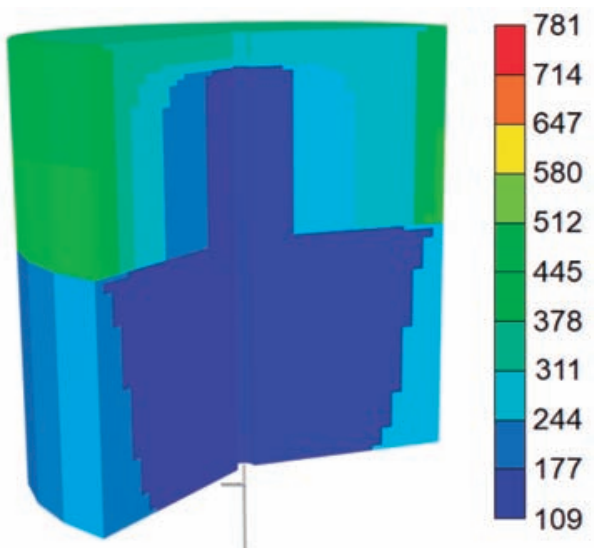

Figura 14. Presión promedio antes del inicio del ciclo 6.

Adicionalmente, debido a los 5 ciclos anteriores de producción, las saturaciones de petróleo han disminuido, principalmente en la zona inferior, donde se dispone de mejores propiedades de roca (Figura 15).

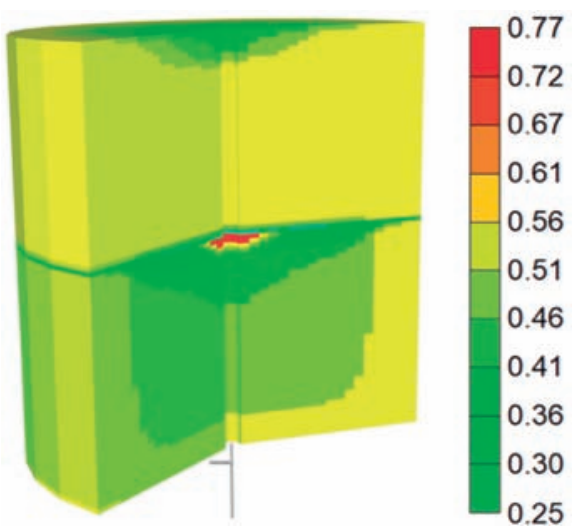

Figura 15. Saturación aceite antes del inicio del ciclo 6. 
Debido al bajo potencial de producción identificado en el ciclo 6, se decidió evaluar la adición de nano partículas en el ciclo 2, el cual en esta etapa temprana del proceso de inyección cíclica de vapor todavía presenta un buen potencial de producción como es evidente en la figura 13.

Los resultados de la inyección de vapor con nano partículas durante el ciclo 2 se presentan en la figura 16. En este caso se estima un incremental de producción de petróleo de 10.963 barriles de crudo comparado con la inyección de solo vapor en el mismo ciclo.

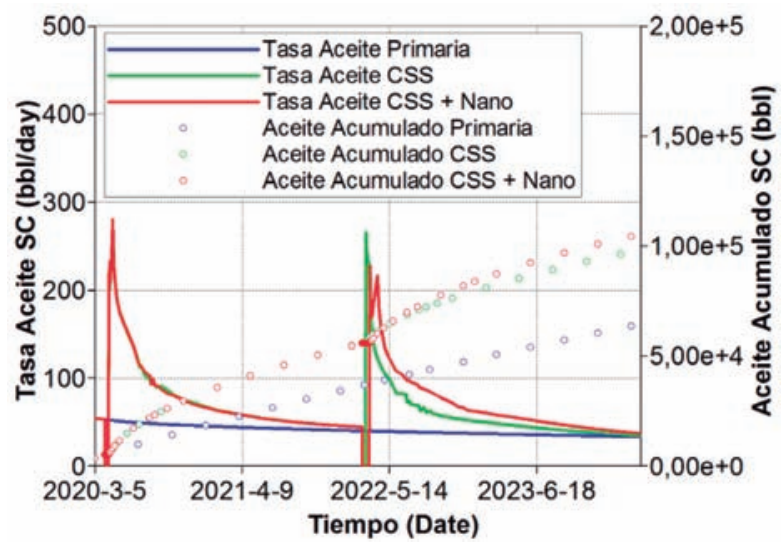

Figura 16. Resultados inyección de vapor con nano partículas (ciclo 2).

Debido a la incertidumbre en las variables utilizadas para simular este proceso, se realizó una sensibilidad a las mismas (Tabla 10). Sin embargo, en ninguno de los casos evaluados se observó una influencia significativa en el recobro incremental de petróleo.

Tabla 10. Sensibilidad parámetros inyección de vapor con nano partículas.

\begin{tabular}{|l|c|c|}
\hline \multicolumn{1}{|c|}{ Parámetro } & Valor inicial & Sensibilidad \\
\hline Ea (BTU/lbmol) & 13.000 & 6.500 \\
\hline Factor de frecuencia (1/día) & 1,8 & $100,1.000 .000$ \\
\hline Temperatura de craqueo, ${ }^{\circ} \mathrm{F}$ & 410 & 300 \\
\hline $\begin{array}{l}\text { Densidad del crudo mejorado } \\
\left.\text { (lb/ft }{ }^{3}\right)\end{array}$ & 57,97 & 50 \\
\hline $\begin{array}{l}\text { Peso molecular del crudo } \\
\text { mejorado (lb/lbmol) }\end{array}$ & 243 & 325 \\
\hline
\end{tabular}

Es importante destacar que estos resultados también pueden estar influenciados por las limitaciones en las capacidades de simular esta tecnología (vapor + nano partículas) con las herramientas disponibles comercialmente. Por lo tanto, se deben hacer mayores esfuerzos para poder incorporar nuevas estrategias que permitan la simulación de procesos híbridos de inyección combinados con nano partículas.

Finalmente, con la revisión de resultados de las tecnologías híbridas se observa que con todas se pueden obtener producciones incrementales; sin embargo, esto depende de las condiciones del yacimiento al momento de su aplicación y la factibilidad económica de cada tecnología.

En el caso de las espumas y el flue gas, los ciclos iniciales, con poco calentamiento del yacimiento, no favorecen su aplicación; pero en ciclos de inyección más avanzados, como el ciclo 6 donde fueron evaluados, si se observan producciones incrementales.

Por otra parte, los solventes y nano partículas generan pobres resultados en el ciclo 6 de inyección, ya que el potencial de producción de fluidos se reduce al disminuir la energía y saturación de aceite en el yacimiento (radio de invasión del pozo). Sin embargo, en ciclos iniciales, su aplicación muestra una mayor eficiencia y respuestas de producción.

Lo anterior indicaría que para mejorar la eficiencia de la inyección cíclica de vapor, sería adecuado incorporar en ciclos iniciales procesos híbridos con solventes o nano partículas, para obtener en etapas tempranas de producción la mayor recuperación de aceite. Mientras que la inyección de vapor con espumas o con flue gas se presentan con mayor aplicabilidad en ciclos de inyección más avanzados. Sin embargo, la aplicación de estos procesos está sujeto a una evaluación económica que permita establecer la viabilidad de su implementación.

\section{Conclusiones}

- La generación de vapor por energía solar puede llegar a suplir total o parcialmente las necesidades de generación de vapor en algunos campos del Magdalena Medio Colombiano (DNI suficiente), ya que el costo de generación y el efecto ambiental es menor. Sin embargo, las inversiones para su implementación son elevadas y el espacio físico requerido para su funcionamiento puede llegar a ser muy grande. Por lo tanto, es necesario hacer una evaluación económica que permita verificar la viabilidad de implementación de esta estrategia de generación de vapor.

- Las tecnologías de calentamiento en fondo resultan de mayor beneficio e impacto en pozos nuevos. Es decir, previo a la aplicación de procesos de inyección 
de vapor, ya que muestra una mejor eficiencia en el uso del calor inyectado durante etapas de mayor presión (energía de empuje) del yacimiento.

- Se identificaron limitaciones en los simuladores comerciales para representar el comportamiento de procesos de inyección hibrida de vapor con otros fluidos (P.E. fenómenos de histéresis y gas atrapado y mecanismos propuestos por el uso de nano partículas). Por lo tanto, se recomienda desarrollar investigaciones detalladas, incluyendo estudios experimentales, que permitan la evaluación numérica más próxima al comportamiento real de estas tecnologías a nivel de laboratorio y campo.

- La inyección de espumas junto con el vapor aumenta el tiempo de duración del ciclo, por lo que se demuestra el efecto divergente que permite el contacto de otras zonas con mayor saturación de petróleo. Su aplicación en ciclos avanzados ( $\geq$ ciclo 6) de la inyección cíclica de vapor puede generar beneficios de producción incremental. Por lo tanto, esta tecnología puede ser considerada para su implementación si la correspondiente evaluación económica es favorable.

- La aplicación de la inyección de vapor con flue gas se presenta como otra opción que genera efectos divergentes (P.E. bloqueo de agua por gas atrapado) para optimizar ciclos avanzados de inyección de vapor. Se estima que la fuente de flue gas puede provenir de los gases de combustión resultados de la generación de vapor. Sin embargo, es necesario realizar una evaluación de los volúmenes de gases de combustión producidos durante la generación de vapor y determinar si su captura y potencial almacenamiento y compresión justifican la economía de este proceso de recobro.

- La simulación numérica del proceso de inyección de vapor con solventes demuestra que la aplicación exitosa de este método depende de la saturación de aceite remanente en el yacimiento. Por lo tanto, se sugiere que su aplicación es recomendada en etapas tempranas de inyección cíclica de vapor. En este estudio se demostró la mayor eficiencia de inyección de vapor con solventes durante el 2do ciclo respecto a su implementación en el 6to ciclo de inyección. A pesar de observarse beneficios de producción incremental, es muy importante al considerar la recuperación del solvente inyectado durante las evaluaciones económicas de esta tecnología.
- El proceso de inyección de vapor con nano partículas es un área de conocimiento naciente y su simulación numérica es un proceso complejo. Basados en el estatus de las herramientas de simulación numérica comercial, aún no se disponen de métodos que permitan representar adecuadamente la diversidad de efectos documentados durante la inyección de vapor con nano partículas.

- De acuerdo a los efectos de la reducción adicional de la viscosidad del aceite durante la inyección de vapor con nano partículas, estudios preliminares de simulación sugieren que este método híbrido de CSS genera mejores beneficios en etapas iniciales de inyección en condiciones de mayores saturaciones de petróleo y potencialmente mayores presiones de yacimiento.

\section{Nomenclatura}

${ }^{\circ} \mathbf{F} \quad$ Grados Farenheit, $1{ }^{\circ} \mathrm{F}=255.9 \mathrm{~K}$

bbl Barriles, $1 \mathrm{bbl}=0.159 \mathrm{~m}^{3}$

BTU British Thermal Unit, 1 BTU $=0.000293$ KWh

BWE Barriles de agua equivalente

$\mathrm{CO}_{2}$ Dióxido de carbono

CSS Inyección cíclica de vapor

d Día

DNI Irradiación Normal Directa

ft $\quad$ Pies; $1 \mathrm{ft}=0.3048 \mathrm{~m}$

$\mathbf{f t}^{3} \quad$ Pies cúbicos, $1 \mathrm{ft}^{3}=0.0283 \mathrm{~m}^{3}$

h Hora

Kh Permeabilidad horizontal

Kv Permeabilidad vertical

KWh Kilo Wattio hora

lb Libras

lbmol Libra mol

$\mathbf{m}^{2} \quad$ Metro cuadrado

mD Mili Darcies

MM Millones

MW Mega Wattio

$\mathrm{N}_{2} \quad$ Nitrógeno

OTSG One Through Steam Generator

Psi Libras por pulgada cuadrada; 1 psi $=6894.7$ Pascales 


$\begin{array}{ll}\mathbf{r} & \text { radio } \\ \text { SI } & \text { Saturación de líquido } \\ \text { Sw } & \text { Saturación de agua } \\ \text { SF } & \text { Inyección continua de vapor } \\ \text { SOR } & \text { Steam Oil Ratio } \\ \text { USD } & \text { Dólares americanos }\end{array}$

\section{Agradecimientos}

Agradecemos a Ecopetrol S.A., especialmente al Instituto Colombiano del Petróleo (ICP) quienes en convenio con la Universidad industrial de Santander por medio del acuerdo Marco No. 5222395 permitieron el desarrollo de la investigación y la publicación de esta.

\section{Referencias Bibliográficas}

1. Ab Kadir, M. Z. A., Rafeeu, Y., \& Adam, N. M. (2010). Prospective scenarios for the full solar energy development in Malaysia. Renewable and Sustainable Energy Reviews, 14(9), 3023-3031.

2. Afzal, S., Ehsani, M. R., Nikookar, M., \& Roayaei, E. (2018). Effect of $\mathrm{Fe} 2 \mathrm{O} 3$ and WO3 nanoparticle on steam injection recovery. Energy Sources, Part A: Recovery, Utilization, and Environmental Effects, 0(3), 251-258.

3. Alomair, O., \& Alajmi, A. (2016, December). Experimental Study for Enhancing Heavy Oil Recovery by Nanofluid Followed by Steam Flooding NFSF. In SPE Heavy Oil Conference and Exhibition. Society of Petroleum Engineers.

4. Ambrosson, F., \& Selin, M. (2016). Solar Concentrating Steam Generation in Alberta, Canada.

5. Andarcia, L., Bermudez, J. M., Reyes, Y., Caycedo, H., \& Suarez, A. F. (2014, September). Potential of Steam Solvent Hybrid Processes in Llanos Basin, Colombia. In SPE Heavy and Extra Heavy Oil Conference: Latin America. Society of Petroleum Engineers.

6. Aya, C. L. D., \& Navarro, S. F. M. (2009). Estudio de la técnica toe to heel steamflood, thsf: una nueva opción para el recobro de crudo pesado. Revista Fuentes, 7(1).
7. Ayatollahi, S., \& Zerafat, M. M. (2012, January). Nanotechnology-assisted EOR techniques: New solutions to old challenges. In SPE international oilfield nanotechnology conference and exhibition. Society of Petroleum Engineers.

8. Bayestehparvin, B., Ali, S. M., \& Abedi, J. (2017, April). Case Histories of Solvent Use in Thermal Recovery. In SPE Western Regional Meeting. Society of Petroleum Engineers.

9. Bruns, F. A., \& Babadagli, T. (2018, April). Recovery Improvement by Chemical Additives to Steam Injection: Identifying Underlying Mechanisms Through Core and Visual Experiments. In SPE Western Regional Meeting. Society of Petroleum Engineers.

10. Cardona Rojas, L. Efecto de nanopartículas en procesos con inyección de vapor a diferentes calidades (Master thesis, Universidad Nacional de Colombia-Sede Medellín).

11. Colina, M. (2009). Evaluación del proceso de inyección alterna de vapor con solventes en pozos horizontales en el yacimiento Jobo 01, campo Jobo 02, a través de la simulación numérica (Doctoral dissertation, Universidad de Oriente).

12. De Ferrer, M. P. (2001). Inyección de agua y gas en yacimientos petrolíferos. Ediciones Astro data SA.

13. Delamaide, E., Cuenca, A., \& Chabert, M. (2016, October). State of the Art Review of the Steam Foam Process. In SPE Latin America and Caribbean Heavy and Extra Heavy Oil Conference. Society of Petroleum Engineers.

14. Díaz, R. J., Navarro, S. F. M., \& Tavera, C. P. S. (2007). Modelo estadístico para la realización de analogías orientadas a procesos de recobro mejorado. Revista Fuentes, 5(1).

15. Franco, C. A., Cardona, L., Lopera, S. H., Mejía, J. M., \& Cortés, F. B. (2016, April). Heavy oil upgrading and enhanced recovery in a continuous steam injection process assisted by nanoparticulated catalysts. In SPE improved oil recovery conference. Society of Petroleum Engineers. 
16. Hamedi Shokrlu, Y. (2013). Enhancement of Heavy Oil/bitumen Thermal Recovery Using Nano Metal Particles (Doctoral dissertation, University of Alberta, Edmonton, Alberta).

17. Hamedi Shokrlu, Y., \& Babadagli, T. (2014). Kinetics of the in-situ upgrading of heavy oil by nickel nanoparticle catalysts and its effect on cyclic-steam-stimulation recovery factor. SPE Reservoir Evaluation \& Engineering, 17(03), 355-364.

18. https://solargis.com/maps-and-gisdata/download/ colombia.

19. Lakhova, A., Petrov, S., Ibragimova, D., Kayukova, G., Safiulina, A., Shinkarev, A., \& Okekwe, R. (2017). Aquathermolysis of heavy oil using nano oxides of metals. Journal of Petroleum Science and Engineering, 153, 385-390.

20. Lesage, D., \& Van de Graaf, T. (2016). Global energy governance in a multipolar world. Routledge.

21. Li, S., Genys, M., Wang, K., \& Torsæter, O. (2015, September). Experimental study of wettability alteration during nanofluid enhanced oil recovery process and its effect on oil recovery. In SPE Reservoir Characterisation and Simulation Conference and Exhibition. Society of Petroleum Engineers.

22. Maity, S. K., Ancheyta, J., \& Marroquín, G. (2010). Catalytic aquathermolysis used for viscosity reduction of heavy crude oils: a review. Energy \& Fuels, 24(5), 2809-2816.

23. Mohammad, A. A., \& Mamora, D. D. (2008, January). Insitu upgrading of heavy oil under steam injection with tetralin and catalyst. In International thermal operations and heavy oil symposium. Society of Petroleum Engineers.

24. Morales, K. A., Patiño, R. R., Navarro, S. F. M., \& Castelblanco, A. X. R. (2015). Uso de un solvente como alternativa para mejorar la inyección cíclica de vapor en un yacimiento de crudo pesado móvil. Revista Fuentes, 13(1), $33-45$.

25. Naranjo, P. A. L., Correa, D. L. B., Navarro, S. F. M., \& Rodriguez, A. O. (2015). Inyección de vapor en medianos. recuperación y rentabilidad. Revista Fuentes, 13(1), 21-31.

26. Palmer, D., \& O’Donnell, J. (2014, March). Construction, Operations and Pernce of the First Enclosed Trough Solar Steam Generation Pilot for EOR Applications. In SPE EOR Conference at Oil and Gas West Asia. Society of Petroleum Engineers.

27. Patel, H., Shah, S., Ahmed, R., \& Ucan, S. (2018). Effects of nanoparticles and temperature on heavy oil viscosity. Journal of Petroleum Science and Engineering, 167, 819-828.

28. Randy, A. (2015, August). Downhole Electrical Heating techniques in the Orinoco Oil Belt, are they always reliable? Appraisal analysis to Petrocedeño's Pilot Project. In SPE Nigeria Annual International Conference and Exhibition. Society of Petroleum Engineers.

29. Rodriguez, R. F., Bashbush, J. L., \& Rincon, A. C. (2008, January). Feasibility of using electrical downhole heaters in Faja heavy oil reservoirs. In International Thermal Operations and Heavy Oil Symposium. Society of Petroleum Engineers.

30. Sandoval Munoz, J. E. (2004). A simulation study of steam and steam-propane injection using a novel smart horizontal producer to enhance oil production (MSc Thesis, Texas A\&M University).

31. Sandrea, R., Dharod, D. (2016). Approach screens reservoir candidates for EOR. Oil \& Gas Journal, April 4, 48-52.

32. SBI Energy (2010). Enhanced Oil Recovery Worldwide, April. www.sbireports.com.

33. Shokrlu, Y. H., \& Babadagli, T. (2014). Viscosity reduction of heavy oil/bitumen using microand nano-metal particles during aqueous and non-aqueous thermal applications. Journal of Petroleum Science and Engineering, 119, 210-220.

34. Sigworth Jr, H. W., Horman, B. W., \& Knowles, C. W. (1983, January). Cogeneration experience in steam EOR applications. In SPE Annual Technical Conference and Exhibition. Society of Petroleum Engineers. 
35. Srinivasan, A., \& Shah, S. N. (2014, October). Surfactant-based fluids containing copper-oxide nanoparticles for heavy oil viscosity reduction. In SPE Annual Technical Conference and Exhibition. Society of Petroleum Engineers.

36. Tajmiri, M., \& Ehsani, M. R. (2016, September). The Potential Of $\mathrm{CuO}$ Nanoparticles to Reduce Viscosity and Alter Wettability at Oil-Wet and Water-Wet Rocks in Heavy Oil Reservoir. In SPE Annual Technical Conference and Exhibition. Society of Petroleum Engineers.

37. Van Heel, A. P., Van Wunnik, J. N., Bentouati, S., \& Terres, R. (2010, January). The impact of daily and seasonal cycles in solar-generated steam on oil recovery. In SPE EOR Conference at Oil \& Gas West Asia. Society of Petroleum Engineers.

38. Villaquirán Vargas, A. P., Rodríguez Castelblanco, A. X., \& Muñoz Navarro, S. F. (2017). Evaluación de la influencia de los gases de combustión en procesos de inyección continua de vapor utilizando generadores de vapor en fondo. Revista ION, 30(2), 65-77.

39. Wei, Y., \& Babadagli, T. (2016, October). Selection of Proper Chemicals to Improve the Pernce of Steam Based Thermal Applications in Sands and Carbonates. In SPE Latin America and Caribbean Heavy and Extra Heavy Oil Conference. Society of Petroleum Engineers.

40. Wei, Y., \& Babadagli, T. (2017, February). Selection of New Generation Chemicals as
Steam Additive for Cost Effective HeavyOil Recovery Applications. In SPE Canada Heavy Oil Technical Conference. Society of Petroleum Engineers.

41. Wen, S., Zhao, Y., Liu, Y., \& Hu, S. (2007, January). A study on catalytic aquathermolysis of heavy crude oil during steam stimulation. In International symposium on oilfield chemistry. Society of Petroleum Engineers.

42. Yegane, M. M., Ayatollahi, S., Bashtani, F., \& Romero, C. (2015, June). Solar generated steam injection in Hamaca, Venezuelan extra heavy oil reservoir; simulation study for oil recovery performance, economical and environmental feasibilities. In EUROPEC 2015. Society of Petroleum Engineers.

43. Yi, S., Babadagli, T., \& Li, H. A. (2016, November). Use of Nickel Nanoparticles for Promoting Aquathermolysis Reaction During Cyclic Steam Stimulation. In International Petroleum Technology Conference. International Petroleum Technology Conference.

44. Zhong, L. G., Liu, Y. J., Fan, H. F., \& Jiang, S. J. (2003, January). Liaohe extra-heavy crude oil underground aquathermolytic treatments using catalyst and hydrogen donors under steam injection conditions. In SPE international improved oil recovery conference in Asia Pacific. Society of Petroleum Engineers.

Recepción: 01 de octubre de 2018

Aceptación: 21 de noviembre de 2018 